\title{
CHAVE DE INTERPRETAÇÃO PARA O MAPEAMENTO DO USO E COBERTURA DA TERRA DA BACIA HIDROGRÁFICA DO ALTO CURSO DO RIO SANTO ANASTÁCIO
}

Carolina Gomes de Melo, Ireli Cristina Luz, Lucas Prado Osco, Ana Paula Marques Ramos.

Universidade do Oeste Paulista - UNOESTE, Faculdade de Engenharia e Arquitetura "Algacyr Maéder Munhóz", Presidente Prudente, São Paulo. E-mail: difonzo.carol@gmail.com.

\section{RESUMO}

No processo de interpretação de imagens, a elaboração e o uso de uma chave de interpretação são essenciais pois facilitam as etapas de identificação e extração de feições em imagens durante a etapa de classificação das mesmas. O objetivo deste trabalho é apresentar uma chave de interpretação elaborada para a extração de feições do uso e cobertura da terra a partir de imagens orbitais. O estudo de caso é a bacia do alto curso do rio Santo Anastácio. Essa bacia contém um reservatório de água que é utilizado para abastecimento público da cidade de Presidente Prudente - SP. O método aplicado na elaboração da chave de interpretação consistiu em combinar conceitos e exemplos sobre diferentes feições da área para a validação dos elementos da chave. Os elementos de interpretação considerados foram a forma ou padrão, a textura, a tonalidade e a localização dos alvos na imagem. Conclui-se que a chave de interpretação deve ser empregada com frequência em estudos envolvendo a extração das feições por imagem, uma vez que auxilia o usuário durante o momento da escolha de feições à serem classificadas. Recomenda-se, ainda, o uso da chave de interpretação em trabalhos que visem atividades de mapeamento para ações de proteção de recursos hídricos e de planejamento de uma determinada região geográfica.

Palavras-chave: chave de interpretação, bacia hidrográfica, produção cartográfica.

\section{INTERPRETATION-KEY FOR THE MAPPING OF USE AND LAND COVER OF THE WATERSHED OF THE HIGHER COURSE OF THE SANTO ANASTÁCIO RIVER}

\begin{abstract}
At the process of image interpretation, the elaboration and the use of an interpretation key are essential because they facilitate the stages of identification and extraction of features in images during the classification stage. The objective of this study is to present an elaborate interpretation key for the extraction of features from the use and land cover of the earth from orbital images. The case study is the upper course of the Santo Anastácio river. This basin contains a reservoir of water that is used for the public supply of the city of Presidente Prudente - SP. The method used in the elaboration of the interpretation key consisted in combining concepts and examples on different features of the area for the validation of the elements of the key. The elements of interpretation considered were the shape or pattern, the texture, the hue and the location of the targets in the image. It is concluded that the interpretation key should be used frequently in studies involving the extraction of the features by image, since it assists the user during the moment of the choice of features to be classified. It is also recommended the use of the interpretation key in works aimed at mapping activities to protect water resources and to plan geographic region activities.
\end{abstract}

Keywords: interpretation key, hydrographic basin, cartographic production. 


\section{INTRODUÇÃO}

O Sensoriamento Remoto é uma ciência que permite a obtenção de informações da superfície terrestre por meio da captação da energia refletida e emitida da superfície, alimentado por tecnologias dos satélites (FLORENZANO ,2011). A confiabilidade e a rapidez no processo de sensoriamento permitem maior facilidade na aquisição dos dados que são importantes para o mapeamento de uso e ocupação do solo de uma determinada área de estudo, sendo uma maneira eficaz e viável de coletar dados para monitorar e modelar uma determinada região, especialmente em países de grande extensão territorial, como o Brasil. Através de softwares de Sistema de Informação Geográfica (SIG), pode-se realizar a classificação de imagens orbitais e a elaboração de mapas temáticos, os quais facilitam a interpretação e análise da qualidade ambiental de uma determinada área.

A interpretação de imagens é um processo pelo qual as "informações são obtidas por técnicas de observação, desenvolvimentos lógicos e acurados, chegando a conclusões (CRUZ, 1981, p. 8). A função da interpretação é caracterizar as feições de interesse, de modo a facilitar a identificação de outras feições com características similares na imagem. Nessa caracterização são definidos os elementos de interpretação, como: tonalidade, forma, textura, estrutura e sombra das feições (FLORENZANO, 2002; NOVO, 2010). Para tanto, são realizadas associações dos objetos por dedução (do geral para o particular), por indução (do particular para o geral) e por analogia, neste momento, relacionamos as propriedades do objeto com seu entorno (NOVO, 2010).

Uma forma de se organizar as informações e auxiliar o usuário na interpretação das feições existentes em uma imagem é a chave de interpretação. A chave consiste na análise visual dos elementos presentes na imagem (tonalidade, cor, textura, forma, tamanho, padrão e outros). Ela é essencial para determinar as categorias temáticas presentes na imagem, tais como mata/cerrado, cultura, pastagem, solo descoberto e água (ROSA, 2009). Por essa razão, a chave de interpretação é uma etapa importante na elaboração de mapas cujo interesse é a representação das feições encontradas em uma determinada imagem, sobretudo em estudos que envolvam o levantamento do uso e cobertura da terra.

\section{OBJETIVO}

O presente trabalho tem por objetivo apresentar a elaboração de uma chave de interpretação para extração de feições de uso e cobertura da terra, a partir de imagens orbitais, do alto curso do rio Santo Anastácio, região do Pontal do Paranapanema.

\section{MÉTODO DE ANÁLISE}

A chave de interpretação proposta neste trabalho atribuiu enfoque às feições de uso e cobertura presentes na bacia do alto curso do rio Santo Anastácio, abrangendo parte dos municípios de Presidente Prudente, Pirapozinho, Regente Feijó, Anhumas e Álvares Machado. A área é tida como importante dentro da bacia do rio Santo Anastácio e, também do Pontal do Paranapanema, pois nesse trecho do rio existe um reservatório em que a água é utilizada para uma parcela do abastecimento público da cidade de Presidente Prudente. Esse reservatório consiste na acumulação da confluência do rio Santo Anastácio com o córrego do Cedro. Na Figura 1 é apresentado a localização geográfica das bacias hidrográficas presentes no Pontal, bem como destacado a área de estudo em cor roxa. 
Figura 1. Localização das bacias hidrográficas do Pontal do Paranapanema.

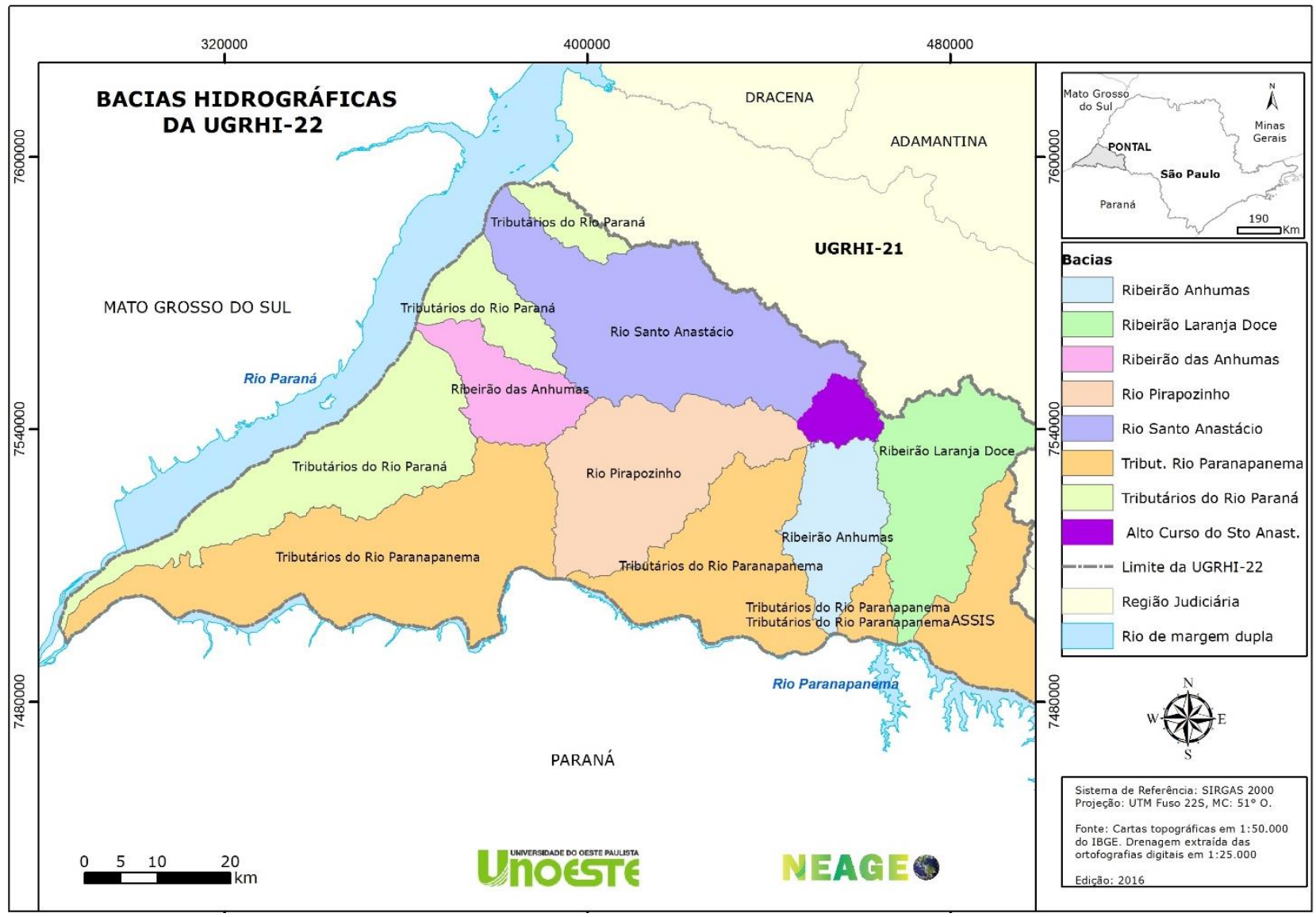

A chave de interpretação sugerida é do tipo seletiva e apoia-se em elementos estabelecidos por autores como Guerra (1993), Calheiros (2004), Novo (2010). A partir dessa literatura conduziram-se estudos para a preparação da chave de interpretação, bem como para a assimilação e explicação de feições de uso e cobertura encontradas na área de estudo. A chave de interpretação proposta neste trabalho, foi obtida através do que (BARIOU, 1978) chama de etapas da chave de interpretação que são: identificação, determinação e interpretação.

A primeira etapa, identificação, representa uma simples leitura da imagem. Nesse momento se utiliza o conhecimento local para a análise da área correlacionando o objeto observado a outro conhecido. Na segunda etapa, determinação, o usuário desenvolve os processos mentais (dedutivos ou indutivos) mesmo que a imagem revele somente uma visão parcial do objeto, como, por exemplo, a copa de uma árvore ou o telhado de uma casa. Finalmente, na interpretação, o usuário cria correlações entre os elementos determinados na imagem e, por fim, elabora hipóteses interpretativas.

A interpretação visual das feições do presente trabalho foi realizada considerando os seguintes elementos definidos para a chave: forma ou padrão, textura, localização e tonalidade. Assim, para facilitar o processo de interpretação, associou-se a esses elementos explicações a respeito das características das feições presentes na imagem aérea da bacia, na composição de bandas falsa cor RGB (654) e composição natural RGB (432).

\section{RESULTADOS E DISCUSSÃO}

No estudo de caso, foram identificadas cinco classes temáticas, sendo: Corpos d'água, Floresta Arbustiva, Floresta Herbácea (pastagem), Área Urbana e Cultura. Adiante encontra-se exposta o Quadro 1 com a chave de interpretação elaborada. 
Quadro 1. Chave de interpretação das feições de uso e cobertura da terra para a bacia hidrográfica do alto curso do rio Santo Anastácio, SP.

\begin{tabular}{|c|c|c|c|c|c|c|c|}
\hline \multirow[b]{2}{*}{$\begin{array}{l}\text { CLASSES } \\
\text { TEMATICA }\end{array}$} & \multicolumn{5}{|c|}{$\begin{array}{c}\text { CHAVE DE INTERPRETAÇÃO } \\
\text { ELEMENTOS DE INTERPRETAÇÃO }\end{array}$} & \multirow{2}{*}{$\begin{array}{c}\text { Composição falsa } \\
\text { Landsat } 8 \\
\text { RGB(654) }\end{array}$} & \multirow{2}{*}{$\begin{array}{c}\begin{array}{c}\text { Composição Natural } \\
\text { Landsat } 8\end{array} \\
\text { RGB(432) }\end{array}$} \\
\hline & DEFINIÇÃO & TEXURA & TONALIDADE & $\begin{array}{c}\text { FORMA } \\
\text { ou } \\
\text { PADRÃO }\end{array}$ & LOCALIZAÇÃO & & \\
\hline \multirow{2}{*}{$\begin{array}{l}\text { Corpos d' } \\
\text { água }\end{array}$} & \multirow{2}{*}{$\begin{array}{l}\text { Corpo d'água } \\
\text { com ou sem } \\
\text { presença de } \\
\text { sedimentos }\end{array}$} & \multirow[b]{2}{*}{ Lisa } & $\begin{array}{l}\text { RGB(654): azul } \\
\text { petróleo }\end{array}$ & \multirow[b]{2}{*}{ Irregular } & \multirow[b]{2}{*}{ Meio rural } & & \\
\hline & & & $\begin{array}{l}\mathrm{RGB}(432) \text { : } \\
\text { verde escuro }\end{array}$ & & & & \\
\hline \multirow[b]{2}{*}{$\begin{array}{l}\text { Área } \\
\text { Urbana }\end{array}$} & \multirow{2}{*}{$\begin{array}{c}\text { Área com } \\
\text { presença } \\
\text { predominante } \\
\text { de construções }\end{array}$} & \multirow[b]{2}{*}{ Rugosa } & RGB(654): rosa & \multirow[b]{2}{*}{ Irregular } & \multirow[b]{2}{*}{ Área Urbana } & & \\
\hline & & & $\begin{array}{c}\text { RGB(432): } \\
\text { Branco com } \\
\text { rosa claro }\end{array}$ & & & & \\
\hline $\begin{array}{l}\text { Estradas e } \\
\text { vicinais }\end{array}$ & $\begin{array}{c}\text { Área } \\
\text { pavimentada } \\
\text { ou não } \\
\text { interconectand } \\
\text { o diferentes } \\
\text { localidades }\end{array}$ & Lisa & $\begin{array}{l}\text { RGB(432): } \\
\quad \text { cinza }\end{array}$ & Linear & Meio rural & & \\
\hline \multirow{2}{*}{$\begin{array}{l}\text { Floresta } \\
\text { arbórea }\end{array}$} & \multirow{2}{*}{ Vegetação } & \multirow{2}{*}{ Rugosa } & $\begin{array}{l}\text { RGB(654): } \\
\text { verde }\end{array}$ & \multirow{2}{*}{ Linear } & \multirow{2}{*}{ Meio rural } & & \\
\hline & & & $\begin{array}{c}\mathrm{RGB}(432): \\
\text { verde escuro }\end{array}$ & & & & \\
\hline \multirow{2}{*}{$\begin{array}{l}\text { Herbácea } \\
\text { Arbustiva }\end{array}$} & \multirow{2}{*}{$\begin{array}{l}\text { Predominante } \\
\text { mente } \\
\text { constituído de } \\
\text { vegetação de } \\
\text { baixo porte }\end{array}$} & \multirow[t]{2}{*}{ Lisa } & $\begin{array}{c}\text { RGB(654): } \\
\text { verde claro } \\
\text { com amarelo }\end{array}$ & \multirow[t]{2}{*}{ Linear } & \multirow[t]{2}{*}{ Meio rural } & & \\
\hline & & & $\begin{array}{l}\text { RGB(432): } \\
\text { verde claro }\end{array}$ & & & & \\
\hline Cultura & $\begin{array}{c}\text { Áreas de } \\
\text { agricultura que }\end{array}$ & Lisa & $\begin{array}{l}\text { RGB(654): } \\
\text { verde claro }\end{array}$ & $\begin{array}{c}\text { Retangula } \\
r\end{array}$ & Meio rural & & \\
\hline
\end{tabular}




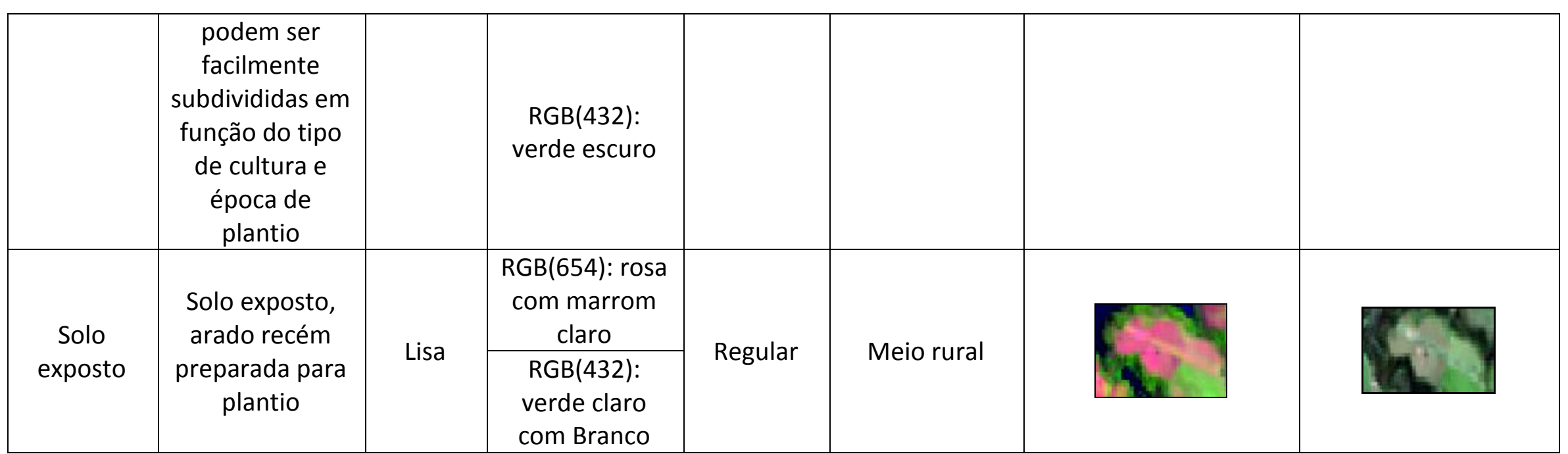


Após a elaboração da chave de interpretação pode-se gerar o produto cartográfico, ou seja, o mapa de uso e cobertura da terra do alto curso do rio, na qual possibilita uma análise sistêmica da área da bacia hidrográfica em estudo. De acordo com o IBGE (2006), os estudos das formas e da dinâmica da ocupação da terra são instrumentos de grande importância para a construção de indicadores ambientais e para a avaliação da capacidade ambiental diante das diversas atividades empregadas na produção, pois o conhecimento adquirido a partir de um mapa de uso e cobertura da terra fornece subsídios para as análises e avaliações dos impactos ambientais.

Figura 2. Mapa de uso e cobertura da terra da bacia hidrográfica do alto curso do rio Santo Anastácio, SP.

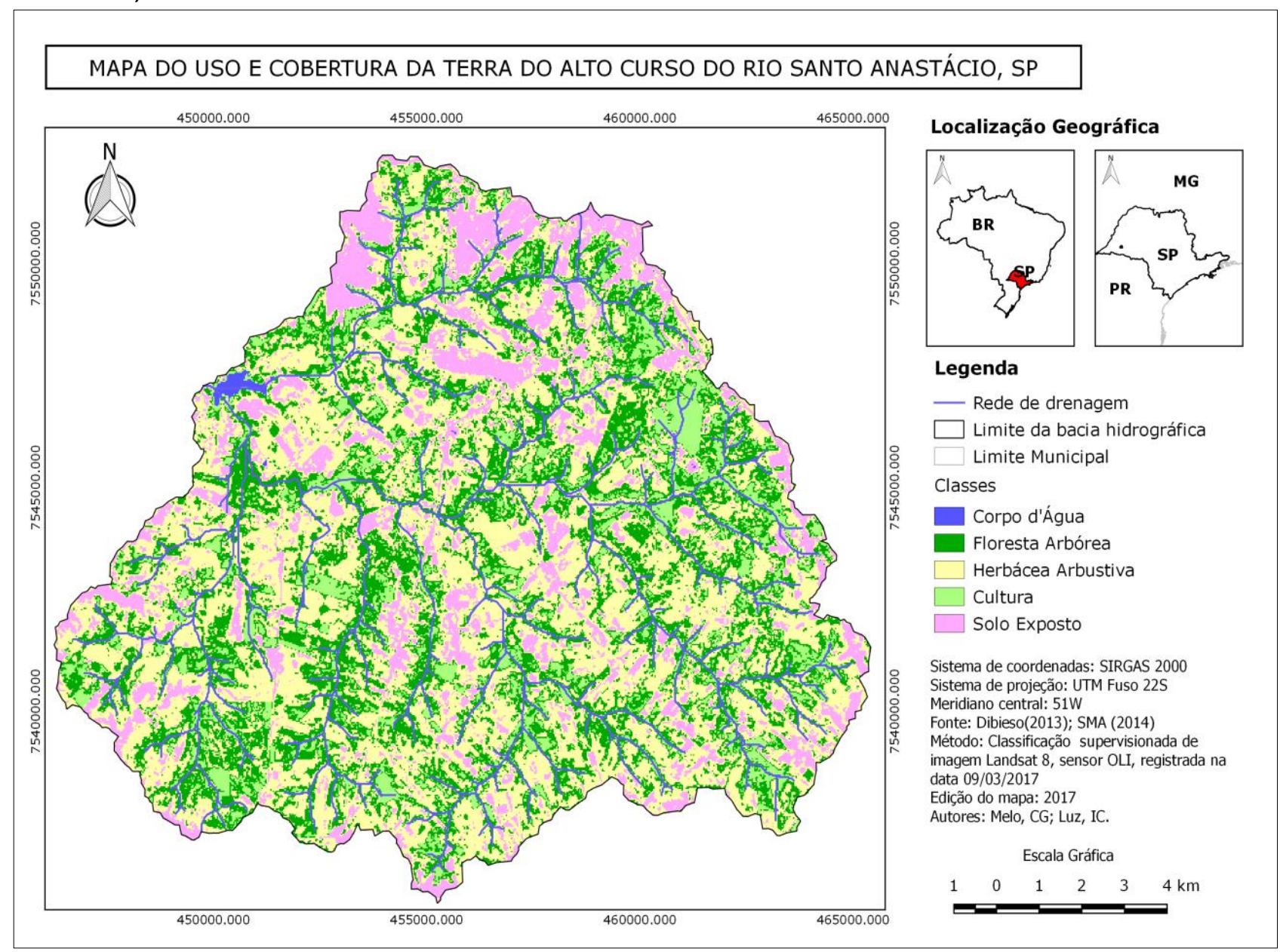

A avaliação quantitativa das classes temáticas representadas no mapa de uso e cobertura é apresentada na Tabela 1. Verificou-se que a cobertura da terra predominante do alto curso do rio Santo Anastácio corresponde a classe herbácea arbustiva (amplamente utilizadas para atividades de pastagem) ocupando cerca de $45,2 \%$ da área total da bacia. Isto se deve a atividade marcante na região que é a pecuária. Para tanto, convém destacar que são necessárias práticas ambientalmente adequadas e de bom manejo para que não ocorram elevadas perdas do solo. 
Tabela 1. Quantificação das classes temáticas do mapa de uso e cobertura da terra elaborado.

\begin{tabular}{c|c|c|c}
\hline Classe & $\begin{array}{c}\text { Extensão da área na } \\
\text { bacia } \mathbf{( m}^{\mathbf{2}} \mathbf{)}\end{array}$ & $\begin{array}{c}\text { Extensão da área na } \\
\text { bacia } \mathbf{( k m}^{\mathbf{2}}\end{array}$ & $\begin{array}{c}\text { Porcentagem da área na } \\
\text { bacia }\end{array}$ \\
\hline Corpo d'água & $475.363,90$ & 0,475 & $0,2 \%$ \\
\hline Floresta Arbórea & $56.348628,7$ & 56,348 & $28,6 \%$ \\
\hline Herbácea Arbustiva & $89.492656,57$ & 89,492 & $45,2 \%$ \\
\hline Cultura & $21.210413,23$ & 21,210 & $10,7 \%$ \\
\hline Solo Exposto & $30.129788,58$ & 30,12 & $15,2 \%$ \\
\hline Total & 197.656851 & 197,656 & $100 \%$ \\
\hline
\end{tabular}

As áreas de Floresta Arbórea abrangem $28,6 \%$ do total da área da bacia, e, na imagem orbital, são observadas ao longo dos percursos dos rios, morros, encostas e área de reserva. $\mathrm{Na}$ classificação não foram separadas as diferentes fisionomias da vegetação nativa encontradas na bacia de estudo, por apresentarem tonalidades e texturas semelhantes na imagem utilizada. Para tanto, o foco do presente estudo de caso não foi o de classificar o tipo de vegetação na bacia, e sim a área de arborização que abrangem principalmente os entornos da bacia.

\section{CONCLUSÃO}

Este trabalho apresentou a elaboração de uma chave de interpretação para a extração de feições de uso e cobertura, a partir de imagens orbitais, no alto curso do rio Santo Anastácio. Conclui-se que a chave auxilia na identificação e reconhecimento de alvos a serem extraídos em imagens de Sensoriamento Remoto. Além disso, a chave de interpretação fornece suporte para o mapeamento de bacia hidrográfica, dentre outros, por viabilizar a organização das feições a serem mapeadas, podendo ser possível quantificação da extensão territorial de cada uma das classes temáticas mapeadas. Esta análise quantitativa é importante para investigar a qualidade ambiental da área em estudo. Tais resultados poderão ser utilizados em outros trabalhos que visem à proteção do meio ambiente.

\section{REFERÊNCIAS}

BARIOU, R. Manuel de télédéction. Paris: Sodipe, 1978.

CALHEIROS, R. de O. Preservação e Recuperação de Nascentes (de água e de vida). Piracicaba, 2004.

CRUZ, O. Alguns conhecimentos básicos para a fotointerpretação. In: AerofotoGeografia 25. São Paulo: IGEOG/USP, 1981.

FLORENZANO, T.G. Imagens de satélite para estudos ambientais. São Paulo: Oficina de Textos, 2002.

FLORENZANO, T.G. Iniciação em Sensoriamento Remoto. 3 ed. São Paulo: Oficina de Textos, 2011. $101 \mathrm{p}$.

GUERRA, A. T. Dicionário geológico, geomorfológico. Rio de Janeiro: Secretaria de Planejamento e Coordenação da Presidência da República, Fundação Instituto Brasileiro de Geografia e Estatística - IBGE, 1993.

IBGE. Manual Técnico de Uso da Terra. 2ae ed., Rio de Janeiro: IBGE, 2006. 
NOVO, E. M. L. M. Sensoriamento remoto: princípios e aplicações. 4. ed. rev. SP: Blucher, 2010.

ROSA, R. Introdução ao sensoriamento remoto. 7ํed., Uberlândia: EDUFU, 2009. 